\title{
Patient and clinician satisfaction with video consultations in dentistry - part one: patient satisfaction
}

\author{
Kate Parker*1 and Matthew Chia'
}

\section{Key points}

\begin{abstract}
Introduction Although often used in medicine, video consultations are less commonly used in dentistry. During the COVID-19 pandemic, the orthodontic team at Croydon University Hospital introduced the use of video consultations to provide continued patient care at a time when face-to-face appointments were not possible.
\end{abstract}

Aim To assess patient and clinician satisfaction with the Attend Anywhere video consultations used by the orthodontic team at Croydon University Hospital.

Method Two separate satisfaction questionnaires were piloted and developed: one for patients and one for clinicians. All patients scheduled for a video consultation between 15 May 2020 and 15 June 2020 were invited to complete a patient satisfaction questionnaire at the end of their consultation.

Results In total, 114 patients attended video consultations in the time period assessed, of which 111 completed a satisfaction questionnaire (97.4\% response rate). Fifty-six percent of patients were female with an average age of 16.5 years. Patient satisfaction was highest for the video consultations being easy to use and convenient. Over $90 \%$ of patients strongly agreed or agreed with all of the satisfaction statements and in terms of whether they would recommend using video consultations. Approximately one-third of patients preferred the video consultation to a faceto-face appointment and one-third of patients were neutral.

Conclusion This study found high levels of patient satisfaction with video consultations in orthodontics. Thoughtful consideration needs to be given to the application of video clinics, going forwards, to ensure that high standards of patient care are maintained.

\section{Introduction}

Video consultations have had an increasing role in medicine; however, their use in dentistry is currently more limited. ${ }^{1}$ They are usually referred to as 'telemedicine' clinics which describe any 'medical service provided remotely via information and communication technology.2

Telemedicine has been shown to have many benefits for both patients and clinicians, which are summarised in Table $1 .^{3,4,5,6,7,8,9}$

${ }^{1}$ Croydon University Hospital, 530 London Road, Thornton Heath, CR7 7YE, UK.

*Correspondence to: Kate Parker

E-mail address: kate.parker3@nhs.net

Refereed Paper.

Accepted 9 September 2020

https://doi.org/10.1038/s41415-021-3007-y
In restorative dentistry, telemedicine has been found to provide safe and effective remote consultations which are equivalent to conventional consultations, with high acceptability reported for both patients and clinicians. ${ }^{10}$ It has been found that the therapeutic presence of the professional is greater for video consultations than for telephone calls and that clinicians and patients communicate in the same way as a face-to-face consultation, with high levels of satisfaction reported. ${ }^{11,12,13,14}$ With the benefits associated with video consultations, it has been suggested that they may replace traditional face-to-face appointments in some areas of medicine. ${ }^{15,16,17}$ The use of telemedicine is being encouraged in dentistry as a result of the challenges of the current healthcare climate. $^{18}$

The use of telemedicine is a new and evolving area. Therefore, it is essential to gain patient and clinician feedback to ensure standards of care are maintained and high levels of satisfaction are achieved. ${ }^{19}$ Existing research shows that patients tend to find web-based communication more acceptable than clinicians..$^{17,20,21,22,23,24}$ Research has also shown that a professional's attitude towards this type of consultation varies according to their previous experience. ${ }^{17,20,21,22,23,24}$ Studies into conventional consultations have shown that the three main areas which contribute to good patient-clinician interactions and high levels of satisfaction are: information exchange; interpersonal relationship building; and shared decisionmaking. ${ }^{25,26,27,28}$ There is currently insufficient evidence on the use of telemedicine in the areas of patient-clinician communication and patient satisfaction comparable to in-person consultations. ${ }^{28}$ 
During the COVID-19 pandemic, the orthodontic team at Croydon University Hospital started to use telemedicine clinics through the Attend Anywhere platform. Within dentistry, there are currently no published studies assessing patient and clinician satisfaction with the use of telemedicine; therefore, this is an essential area of new research.

\section{Aims}

This service evaluation aimed to assess patient and clinician satisfaction with the Attend Anywhere telemedicine clinics used by the orthodontic team at Croydon University Hospital. The focus of this article is patient satisfaction. Part two of this paper focuses on clinician satisfaction.

\section{Method}

Two questionnaires were used to assess satisfaction: one for patients and the other for clinicians. Both questionnaires were specifically developed for this study and were piloted and modified before use. Both surveys consisted of nine questions, with the majority focusing on the same topic areas to assess patient and clinician satisfaction with the same telemedicine clinic interaction.

For the patient survey, the first two questions asked about patients' gender and age. The next seven questions assessed the patient's experience of the video consultation and asked patients to rate seven statements using a five-point Likert scale (strongly agree, agree, neither agree nor disagree, disagree, strongly disagree). ${ }^{29}$ Patients were asked to rate the following statements:

- The video consultation was easy to use

- The video consultation was convenient

- I was able to talk about my care easily

- Using the video consultation has been a positive experience

- I would use a video consultation again

- I would recommend using video consultations to other people

- Where possible, I would prefer to use a video consultation instead of a face-to-face appointment.

Following this, there was a free-text section for comments and suggestions.

One hundred and twenty-eight consecutive patients scheduled for an appointment with the orthodontic team between 15 May 2020 and

Table 1 The benefits of telemedicine for clinicians and patients reproduced with permission from K. Parker and M. Chia, 'Remote working in dentistry in a time of crisis tools and their uses', Dental Update, $2020^{3}$

\begin{tabular}{l|l} 
Clinician & Patient \\
\hline Improved clinical networks & Limiting the risk and spread of infection \\
\hline Increased quality of services & Minimising travel \\
\hline Develop communication with patients & Improved access to care \\
\hline Reduced patient non-attendance & Reducing stress \\
\hline Cost effective and efficient & Promoting self-care and prevention \\
\hline Reduced administrative workload & Enhanced communication and convenience \\
\hline Good experience and satisfaction & Good experience and satisfaction \\
\hline
\end{tabular}

Fig. 1 The video consultation was easy to use

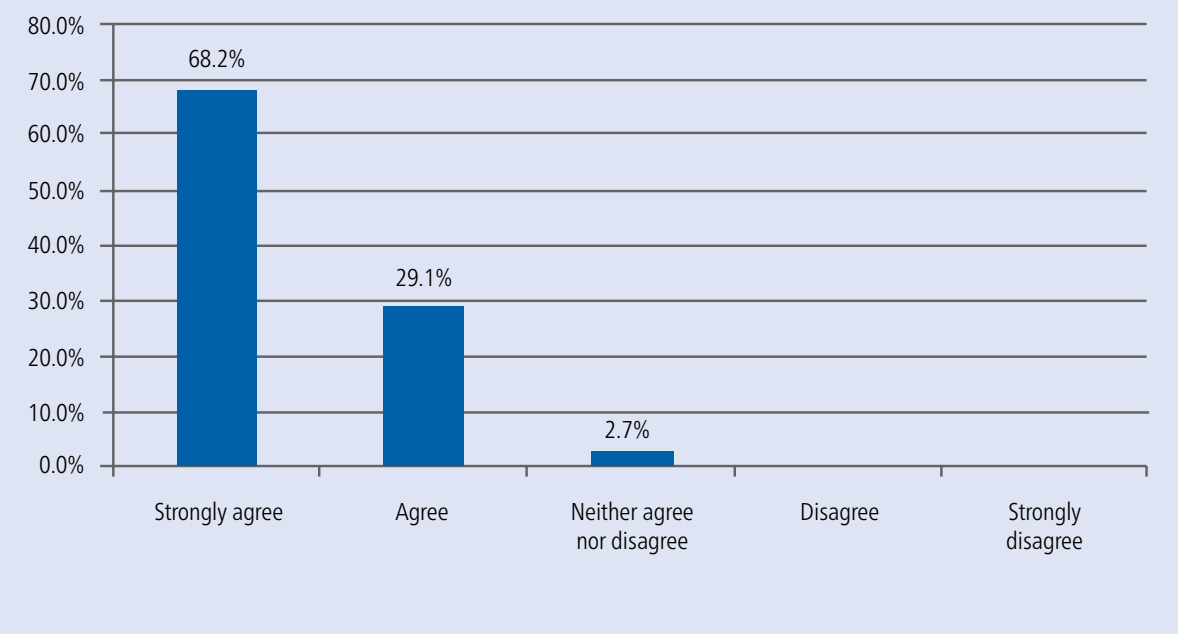

Fig. 2 The video consultation was convenient

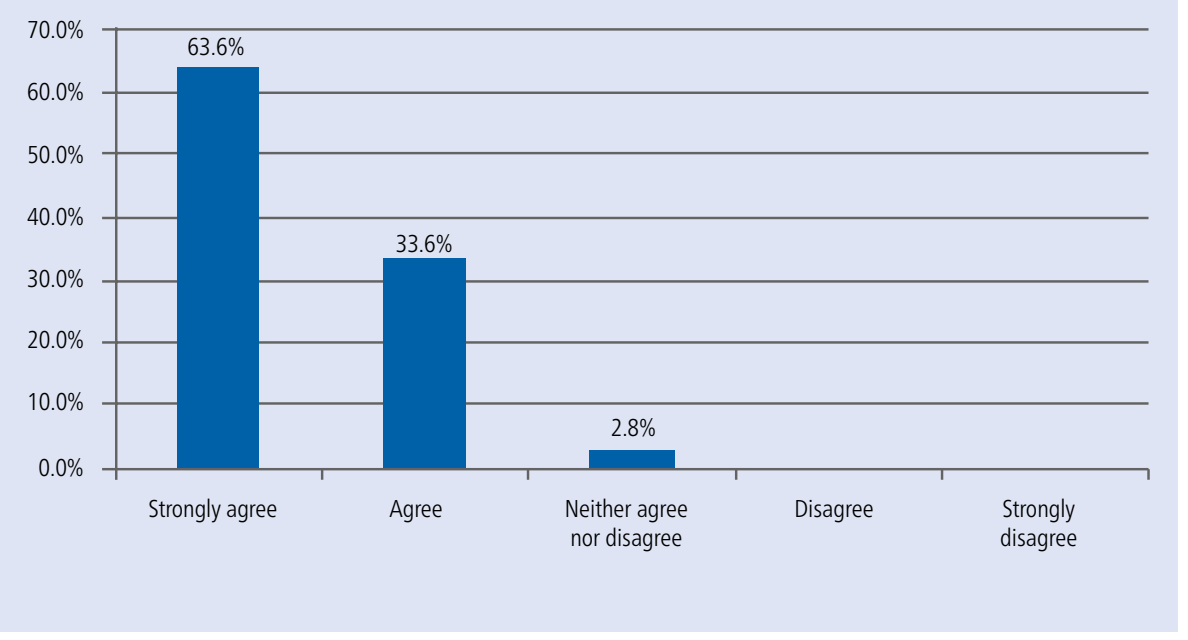

15 June 2020 were contacted by telephone to offer them a video consultation to replace their planned face-to-face appointment, due to the COVID-19 pandemic. Of these patients, eight declined a video consultation and were scheduled instead for a telephone consultation. A further six patients failed to 'attend' their video consultation. This resulted in 114 patients being seen for a video consultation between 15 May 2020 and 15 June 2020. The video consultations were carried out with the clinician who was 


\section{Fig. 3 I was able to talk about my care easily}

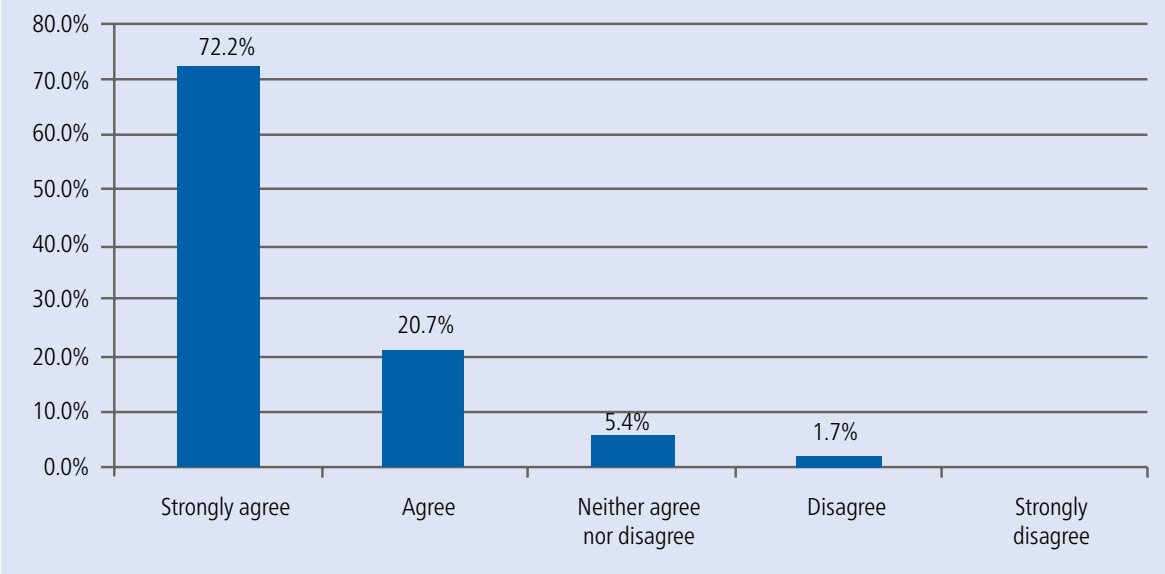

Fig. 4 The video consultation has been a positive experience

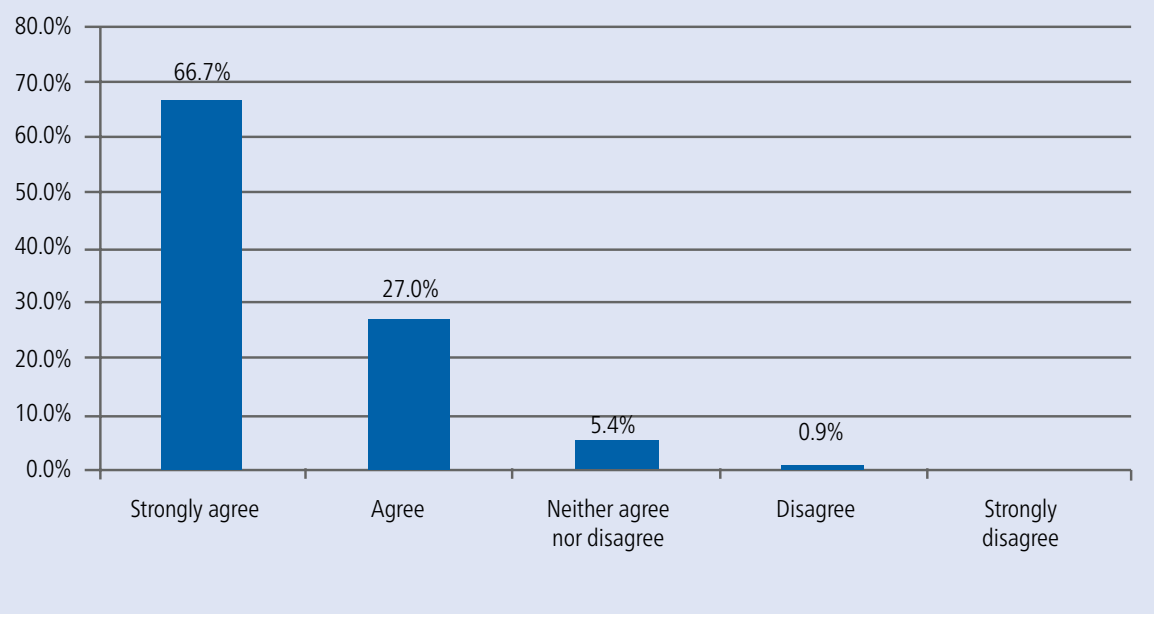

Fig. 5 I would use the video consultation again

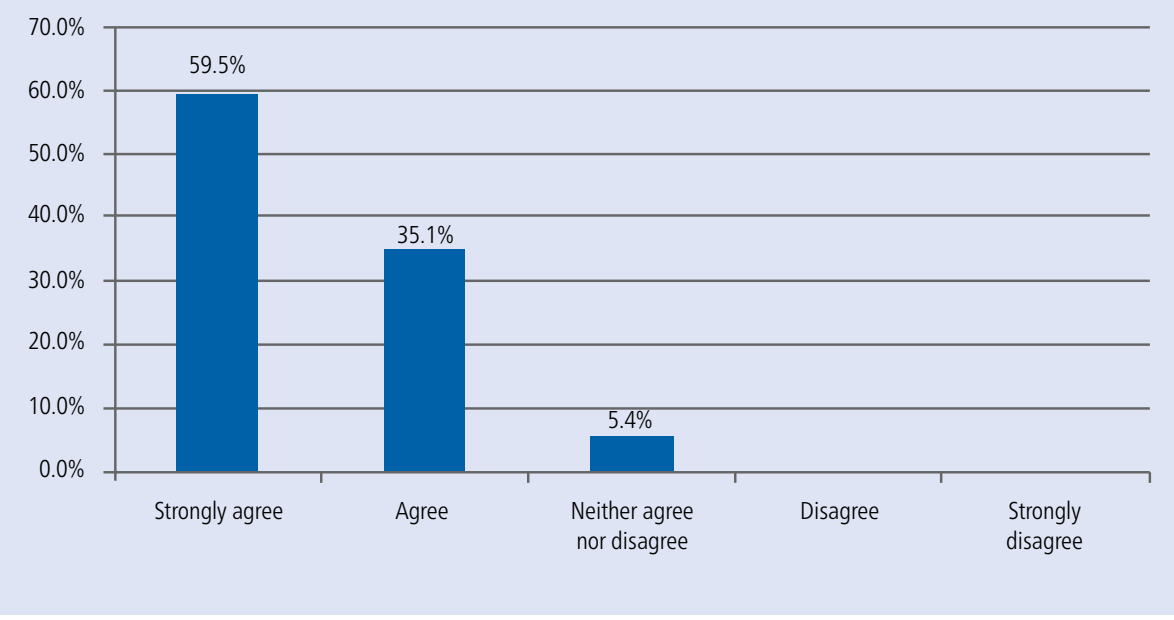

providing the patient's care and a parent if the patient was under the age of 18 years old or if the patient desired a parent to be present. Consent was obtained from the patient
All patients were included in the study irrespective of appointment type, the reason for the consultation and which clinician they were seen by. This ensured a large cross section of patients were included in the study that represented the population seen by the orthodontic team in the hospital.

At the end of every video consultation, the patient was invited to complete the satisfaction questionnaire. They were advised that the survey was confidential, voluntary and would not affect their ongoing care. When the video consultation ended, the survey would automatically display on the patient's screen for completion. The following day, the administrative team sent a reminder e-mail to all patients with a link to the questionnaire; inviting patients to complete the questionnaire if they had not already done so. The questionnaire was primarily completed by the patient with assistance from a parent who had also attended the video consultation, if required.

The questionnaire was hosted by Survey Monkey which collated the data anonymously. Data were then analysed using Survey Monkey and Microsoft Excel.

\section{Results}

Between 15 May 2020 and 15 June 2020, of the 114 patients who had video consultations, 111 patients completed the satisfaction questionnaire (97.4\% response rate). Of the patients who completed the questionnaire, $58.6 \%$ were female and $41.4 \%$ were male, with a mean age of 16.5 years (range: $9-50$ years). The 'failure to attend' rate for video consultations was $5.3 \%$ (six patients).

The results for the seven satisfaction statements are shown in Figures 1, 2, 3, 4, 5, 6 and 7. Figure 1 shows if patients found the video consultation easy to use, and it can be seen that $68.2 \%$ of patients strongly agreed and $29.1 \%$ agreed.

Figure 2 shows that the majority of patients strongly agreed that the video consultation was convenient $(63.6 \%)$ and another third of patients agreed (33.6\%).

Figure 3 shows that the majority of patients felt that they could talk about their care easily, with $92.9 \%$ either strongly agreeing or agreeing with this statement.

The majority of patients either strongly agreed $(66.7 \%)$ or agreed $(27.0 \%)$ that the video consultation was a positive experience, and similarly, most patients strongly agreed (59.5\%) or agreed (35.1\%) that they would use the video consultation again (Figures 4 and 5). 
When patients were asked if they would recommend using video consultations to other people, the majority of patients strongly agreed $(58.6 \%)$ or agreed (31.5\%) (Fig. 6).

The results for whether patients would prefer a video consultation to a face-to-face appointment were more evenly spread across the different ratings, with approximately onethird of patients strongly agreeing or agreeing (33.3\%), one-third neither agreeing nor disagreeing (32.4\%) and one-third disagreeing or strongly disagreeing (34.3\%) (Fig. 7).

At the end of the questionnaire, patients could add free-text comments. A selection representing the full range of patient comments is shown in Box 1. Overall, the remarks were positive, with patients finding the video consultations easy to use and helpful as an alternative to conventional appointments. The comments also reflect that some patients experienced technical difficulties and understood the disadvantages of video consultations.

\section{Discussion}

This study establishes high levels of patient satisfaction with telemedicine clinics for orthodontic patients across a broad range of appointment types. For all of the six satisfaction statements, the vast majority of patients either strongly agreed or agreed, with only a small number neither agreeing nor disagreeing. For only two statements did a very small number of patients disagree.

The areas with the highest satisfaction were ease of use and convenience, both of which had over $97 \%$ of patients strongly agreeing or agreeing. The high degrees of satisfaction in the ease of use demonstrate that telemedicine is technologically accessible and only requires basic computer literacy. This is relevant in ensuring that this mode of communication is available to all demographics of society. The benefit of convenience for patients is also highlighted in the patient comments. Even though a set appointment time was given to patients, the convenience was emphasised by not having to travel and the patient attending the video consultation from a familiar, comfortable environment.

For the remaining satisfaction statements, more than $90 \%$ of patients strongly agreed or agreed. The area with the relatively lowest satisfaction was the ability of patients to talk about their care easily, with $92.8 \%$ of patients

\section{Fig. 6 I would recommend using video consultations to other people}

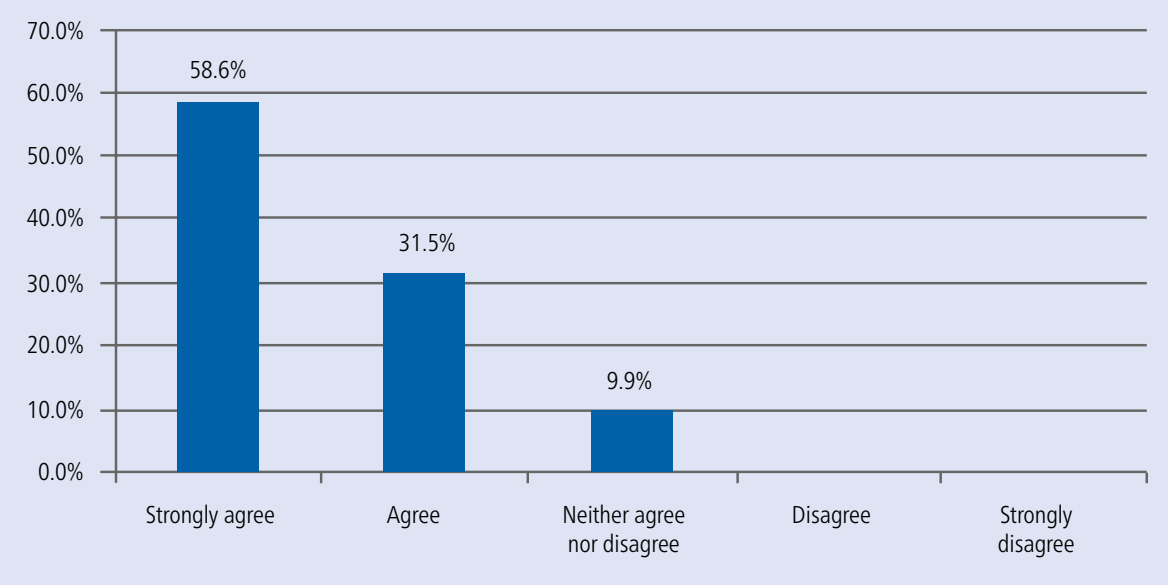

Fig. 7 Where possible, I would prefer to use a video consultation instead of a face-to-face appointment

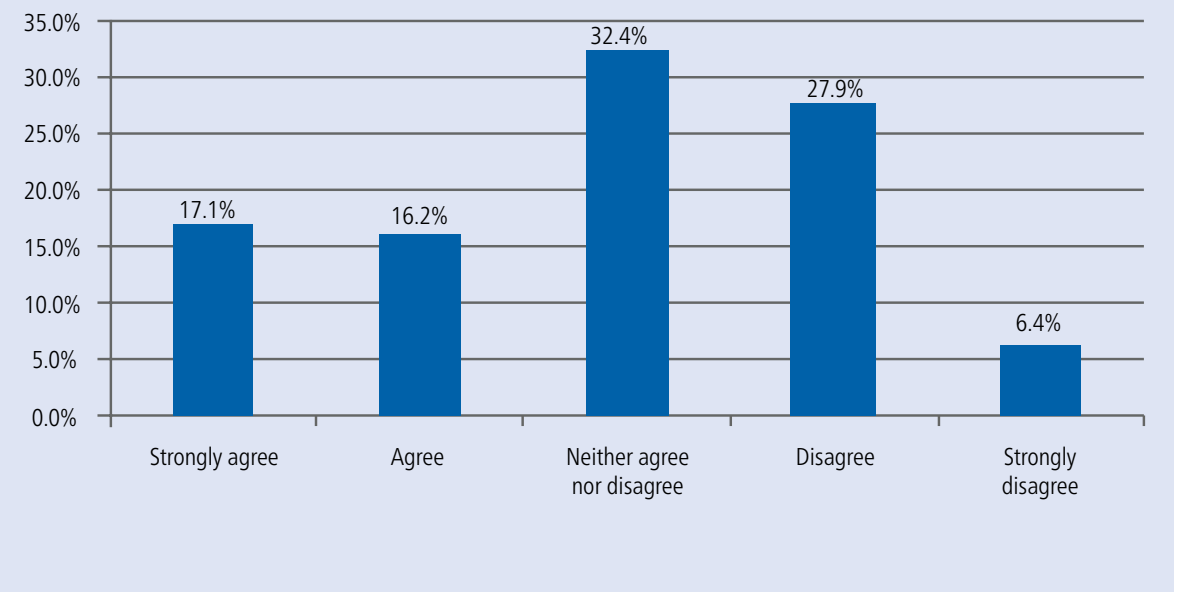

strongly agreeing or agreeing. However, this category still scored well. This score may also reflect the technical difficulties experienced by patients and adjusting to this new way of communicating. Considering that 55\% of communication is with facial expression and body language, this may explain how verbal communication is enhanced by the visual element from the video. ${ }^{30}$ This is significant for patients whose first language may not be English or who have hearing impairments.

The final question asked patients if they would prefer a video consultation to a face-to-face appointment, if appropriate. This statement had the widest range of responses. Approximately one-third of patients strongly agreed or agreed, one-third neither agreed nor disagreed and one-third of patients disagreed or strongly disagreed. Further observations can be made when assessing the responses to this question in conjunction with the patient comments. The patients who reported a preference for face-to-face consultations commented on issues such as poor internet connection, an inability to have mechanical treatment completed and an underlying reassurance they felt when seeing a clinician in person. Conversely, patients who preferred the video consultations cited aspects such as improved convenience, good-quality audio visuals and preferring not to travel to appointments. This gives qualitative data and more insight into the basis of patient satisfaction with video consultations. There are multiple factors that may contribute to this patient bias, including experience with video consultations or social media, interactions with healthcare professionals, treatment progress, technological barriers or ease of attending appointments (with travelling, scheduling or personal circumstances). 


\section{Box 1 A selection of the patient comments about their video consultation}

- Very accessible and well organised

- It was great! Very easy to use and set up and I didn't have to wait long to be seen

- It was good to be able to see my orthodontist on the screen rather than just talking on the phone

- Worked well. Thank you

- The doctor was able to review my teeth and my retainer efficiently

- Seamless experience and a lot more time efficient for me. Thank you!

- Convenient for feedback using this system but will eventually need a face-to-face consultation

- The video consultation worked really well and at this point in time feels safer than coming into the hospital. Thank you very much for taking the time to check on my son. Highly recommend the video calls

- All ok and easy to use; however, it was very difficult to show inside the mouth/braces/gaps between teeth. Looking forward to traditional consultations for these reasons

- It took a few goes to work and the waiting was a bit long, the music was annoying. But overall a positive experience

- It's a good way for keeping patients and staff updated for quick check-ups

- The call was a success and would not hesitate to use it again providing no manual treatment is required

- I found the video consultation super easy to use and very convenient. I feel more optimistic and encouraged

- Sometimes the call was a bit laggy. The doctor was talking but there was a delay with the sound coming through

- Very useful and reassuring to be able to communicate with your consultant. However, 1-1 contact cannot be beaten.

Further studies to assess this area in more detail would be beneficial.

Overall, the high levels of agreement with the areas of positive experience, using the video consultation again and recommending video consultations all provide strong evidence for the future use of this technology. Additionally, the fact that approximately two-thirds of patients agreed with or were neutral towards telemedicine indicates that this is an acceptable and viable option of communication for patients in dentistry.

The low number of patients that 'did not attend' their video consultations illustrates another advantage of telemedicine in improving attendance and access to care. The 5.3\% 'failure to attend' rate compares favourably to $23 \%$ non-attendance rates across medical specialties for face-to-face appointments. ${ }^{31}$

For this study, the response rate was excellent and higher than would normally be expected for a questionnaire-based study. ${ }^{32}$ The high response rate may be due to the survey appearing immediately on the patient's own screen after the consultation, making it convenient and easy to complete. Additionally, the questionnaire was short with only nine questions, contributing to low respondent fatigue. ${ }^{33}$ With a high response rate, the results are likely to be accurate and precise in reflecting the views of the orthodontic patients within the department. All consecutive patients were included, with no exclusions, which further makes the results applicable across a range of different patient ages, clinic types and care given.

Our profession has a practical nature to the assessments and treatments provided compared to some medical specialties. Hence, the use of telemedicine will always have its limitations and will never be able to fully replace face-toface appointments. However, there are certain areas where it may be utilised. In orthodontics, for example, these may be retainer reviews, removable and functional appliance reviews, and monitoring dental development, where clinicians feel comfortable, have an existing relationship with their patients and are able to provide a high standard of care without the need to see the patient in person at every appointment.

This study concentrates on orthodontic patients, but some inferences can be extended to other patient groups in dentistry due to the high response rate and the generic nature of the patient experiences. More studies are needed to gain precise information on other groups and if they report similar levels of satisfaction. However, the conclusive and compelling data for this cohort of patients provide a strong foundation and catalyst for future work on video consultations within other fields of dentistry.

\section{Conclusion}

As telemedicine in dentistry is a developing area of communication, this study provides evidence for patient confidence and acceptance of this technology, particularly with video consultations in orthodontics. This is demonstrated by the high levels of patient satisfaction. However, thoughtful consideration needs to be given to the application of telemedicine to ensure that high standards of patient care are maintained. This should be explored more in dentistry, with these results forming a basis for further research.

\section{Conflict of interest}

The authors declare no conflicts of interest.

\section{Acknowledgements}

The authors would like to thank: Julia Radecki, Renu George, Sara Stephens and Varlie Bacon for their assistance with this project.

\section{References}

1. Kreps G L, Neuhauser L. New directions in eHealth communication: Opportunities and challenges. Patient Educ Couns 2010; 78: 329-336.

2. General Medical Council. Regulatory Approaches to Telemedicine. Available at https://www.gmc-uk.org/ about/what-we-do-and-why/data-and-research/ research-and-insight-archive/regulatory-approachesto-telemedicine (accessed May 2020).

3. Parker K, Chia M. Remote working in dentistry in a time of crisis - tools and their uses. Dent Update 2020; 47: 515-526.

4. ezTalks. Top 6 Benefits of Video Conferencing in Healthcare. Available at https://www.eztalks.com/ video-conference/benefits-of-video-conferencing-inhealthcare.html (accessed May 2020).

5. Mega Meeting. The Modern House Call: The Benefits of Video Conferencing in Healthcare. 2019. Available at https://www.megameeting.com/news/benefits-ofvideo-conferencing-in-healthcare/ (accessed May 2020).

6. GP. How Video Consultations can benefit Patients and the NHS. 2016. Available at https://www.gponline. com/video-consultations-benefit-patients-nhs/ article/1401346 (accessed May 2020).

7. Donaghy E, Atherton $\mathrm{H}$, Hammersley $\mathrm{V}$ et al. Acceptability, benefits, and challenges of video consulting: A qualitative study in primary care. $\mathrm{Br} J \mathrm{Gen}$ Pract 2019; D0I: 10.3399/bjgp19X704141.

8. Shaw S, Wherton J, Vijayaraghavan $S$ et al. Advantages and limitations of virtual online consultations in a NHS acute trust: the VOCAL mixed-methods study. Southampton: NIHR Journals Library, 2018.

9. NHS England. GP Online Services: the key benefits Available at https://www.england.nhs.uk/gp-onlineservices/learning-so-far/key-benefits/ (accessed May 2020)

10. Martin N, Shahrbaf S, Towers A, Stokes C, Storey C Remote clinical consultations in restorative dentistry: a clinical service evaluation study. Br Dent J 2020; 228: 441-447.

11. Greenhalgh T, Koh G C H, Car J. Covid-19: A Remote Assessment in Primary Care. BMJ 2020; DOI: 10.1136/ bmj.m1182.

12. Yeung A, Johnson D P, Trinh N-H, Weng W-C C, Kvedar J, Fava M. Feasibility and effectiveness of telepsychiatry services for Chinese immigrants in a nursing home. Telemed J E Health 2009; 15: 336-341. 
13. Wade V, Whittaker F, Hamlyn J. An evaluation of the benefits and challenges of video consulting between general practitioners and residential aged care facilities. J Telemed Telecare 2015; 21: 490-493.

14. Guan W-J, Ni Z-Y, Hu Y et al. Clinical Characteristics of Coronavirus Disease 2019 in China. N Engl J Med 2020 382: 1708-1720.

15. Knight P, Bonney A, Teuss $G$ et al. Positive clinical outcomes are synergistic with positive educational outcomes when using telehealth consulting in general practice: a mixed-methods study. J Med Internet Res 2016; DOI: 10.2196/jmir.4510

16. Weiner J P Yeh S, Blumenthal D. The impact of health information technology and e-health on the future demand for physician services. Health Aff (Millwood) 2013: 32: 1998-2004

17. Haluza D, Jungwirth D. ICT and the future of health care: aspects of health promotion. Int J Med Inform 2015; 84: 48-57.

18. Hurley $S$. Why re-invent the wheel if you've run out of road? Br Dent J 2020; 228: 755-756.

19. Al-Abri R, Al-Balushi A. Patient satisfaction survey as a tool towards quality improvement. Oman Med J 2014: 29: 3-7.

20. Peeters J M, Krijgsman J W, Brabers A E, Jong J D, Friele $R D$. Use and uptake of eHealth in general practice: $a$ cross-sectional survey and focus group study among health care users and general practitioners. JMIR Med Inform 2016; DOI: 10.2196/medinform. 4515.

21. Ariens L F, Schussler-Raymakers F M, Frima C et al. Barriers and facilitators to eHealth use in daily practice: perspectives of patients and professionals in Dermatology. J Med Internet Res 2017; DOI: 10.2196/ jmir.7512.

22. Ruiz Morilla M D, Sans M, Casasa A, Giménez N Implementing technology in healthcare: Insights from physicians. BMC Med Inform Decis Mak 2017; 17: 92.

23. Lupiáñez-Villanueva F, Hardey M, Torrent J, Ficapal P. The integration of Information and Communication Technology into medical practice. Int J Med Inform 2010; 79: 478-491.

24. Illiger K, Hupka M, von Jan U, Wichelhaus D, Albrecht U. Mobile technologies: Expectancy, usage, and acceptance of clinical staff and patients at a university medical centre. JMIR Mhealth Uhealth 2014; DOI: 10.2196/mhealth.3799.

25. Ong L M, De Haes J C, Hoos A M, Lammes F B. Doctorpatient communication: A review of the literature. Soc Sci Med 1995; 40: 903-918.

26. Bensing J, van Dulmen S, Tates K. Communication in context: New directions in communication research. Patient Educ Couns 2003; 50: 27-32.
27. de Haes $\mathrm{H}$, Bensing J. Endpoints in medical communication research, proposing a framework of functions and outcomes. Patient Educ Couns 2009; 74: 287-294.

28. Tates K, Antheunis M L, Kanters S, Nieboer T E, Gerritse M B. The Effect of Screen-to-Screen Versus Face-to-Face Consultation on Doctor-Patient Communication: An Experimental Study with Simulated Patients. J Med Internet Res 2017; DOI: 10.2196/jmir.8033.

29. Likert R. A technique for the measurement of attitudes. Arch Psychol 1932; 22: 1-55.

30. Mehrabian A. Nonverbal communication. New Brunswick: Aldine Transaction, 1972.

31. Dantas L F, Fleck J L, Oliveira F L, Hamacher S. No-shows in appointment scheduling - A systematic literature review. Health Policy 2018; 122: 412-421.

32. Horevoorts N J, Vissers P A, Mols F, Thong M S, van de Poll-Franse $L V$. Response rates for patientreported outcomes using web-based versus paper questionnaires: comparison of two invitational methods in older colorectal cancer patients. J Med Internet Res 2015; DOI: 10.2196/jmir.3741.

33. Ben-Nun P. Respondent fatigue. In Lavrakas P J (ed) Encyclopaedia of Survey Research Methods. pp 742-743. Thousand Oaks: SAGE Publications Inc, 2008. 